\title{
IRIS BIOMETRIC RECOGNITION SYSTEM EMPLOYING CANNY OPERATOR
}

\author{
Binsu C. Kovoor ${ }^{1}$, Supriya M.H. ${ }^{2}$ and K. Poulose Jacob ${ }^{3}$ \\ ${ }^{1,3}$ Department of Computer Science, Cochin University of Science and \\ Technology, Cochin, Kerala, India \\ ${ }^{1}$ binsu. kovooregmail.com., ${ }^{3} \mathrm{kpj}$ ecusat.ac. in \\ ${ }^{2}$ Department of Electronics, Cochin University of Science and Technology, \\ Cochin, Kerala, India \\ supriyadoe@gmail.com
}

\begin{abstract}
Biometrics has become important in security applications. In comparison with many other biometric features, iris recognition has very high recognition accuracy because it depends on iris which is located in a place that still stable throughout human life and the probability to find two identical iris's is close to zero. The identification system consists of several stages including segmentation stage which is the most serious and critical one. The current segmentation methods still have limitation in localizing the iris due to circular shape consideration of the pupil. In this research, Daugman method is done to investigate the segmentation techniques. Eyelid detection is another step that has been included in this study as a part of segmentation stage to localize the iris accurately and remove unwanted area that might be included. The obtained iris region is encoded using haar wavelets to construct the iris code, which contains the most discriminating feature in the iris pattern. Hamming distance is used for comparison of iris templates in the recognition stage. The dataset which is used for the study is UBIRIS database. A comparative study of different edge detector operator is performed. It is observed that canny operator is best suited to extract most of the edges to generate the iris code for comparison. Recognition rate of $89 \%$ and rejection rate of $95 \%$ is achieved.
\end{abstract}

\section{KEYWORDS}

Iris, Canny, Daugman, wavelet, Hamming Distance.

\section{INTRODUCTION}

Biometrics-based personal authentication systems have recently gained intensive research interest due to the unreliability and inconvenience of traditional authentication systems. Biometrics recently became a vital element of any successful person identification solutions as biometric traits cannot be stolen, shared or even forgotten[1]. Among biometric technologies, iris-based authentication systems bear more advantages than any other biometric technologies as it offers an excellent recognition performance. Iris patterns are believed to be unique due to the complexity of the underlying environmental and genetic processes that influence the generation of iris pattern. These factors result in extraordinary textural patterns that are unique to each eye of an individual and is even distinct between twins[2]. 
Iris, a delicate circular diaphragm, lies between the cornea and the lens of the human eye. Since the iris patterns do not alter significantly during a person's lifetime, it is considered to be one of the most stable and precise personal identification biometric [1][3][4]. Iris recognition techniques identifies a person by mathematically analyzing the unique random patterns that are visible within the iris and making comparisons with an already existing database.

The basic block diagram of iris recognition system is as shown in Figure 1. The different stages in the implementation of the system consist of segmentation, normalization, feature extraction and matching.

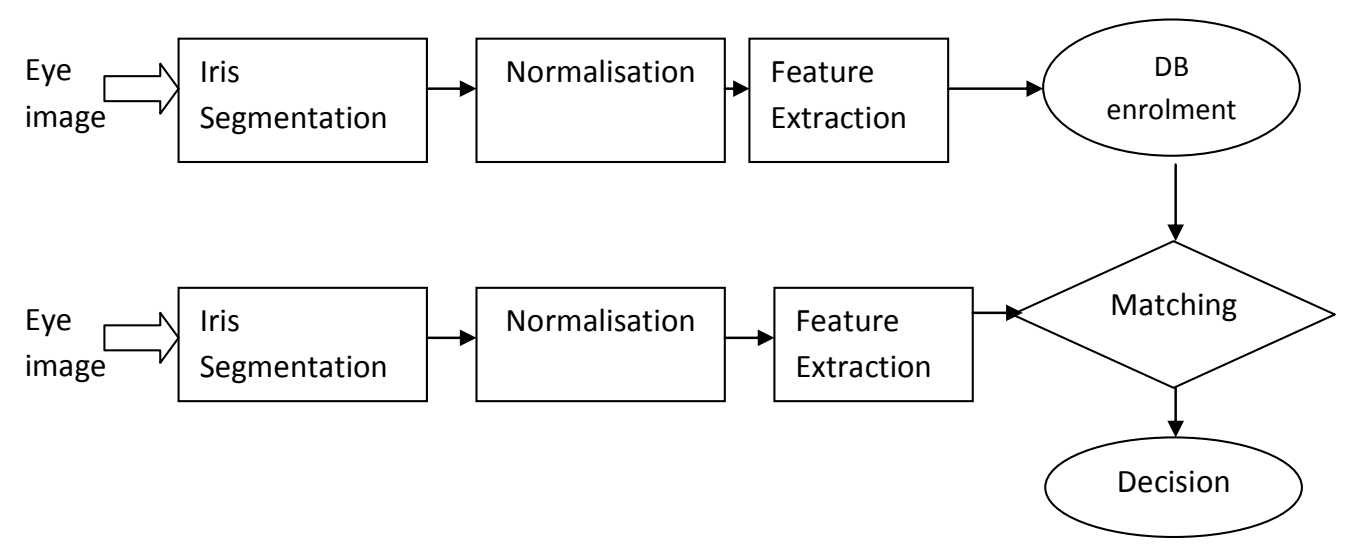

Figure 1. Basic block diagram of iris recognition System

A prototype has been implemented and tested using UBIRIS database[5]. The prototype had a recognition rate of $89 \%$ and rejection rate of $95 \%$.

\section{MethodologY}

The pre-processing stage requires the localization of the iris which generally involves the detection of the edge of the iris as well as that of the pupil. Since varying levels of illumination can result in dimensional inconsistencies between eye images due to the stretching of the iris, normalization needs to be performed so that iris region is transformed to have fixed dimensions. After unwrapping the normalized iris region into a rectangular region, it is encoded using Haar wavelets to generate the iris code. In the recognition stage, Hamming distance is used for comparison of the iris code, the most discriminating feature of the iris pattern, with the existing iris templates.

\subsection{Iris Localization}

The different steps involved in the Iris Segmentation or localization is as shown in Figure 2. The iris region approximated by a ring defined by the iris/sclera (limbic) boundary and the iris/pupil (pupillary) boundary, needs isolation by the removal of the eyelids and eyelashes [6]. 


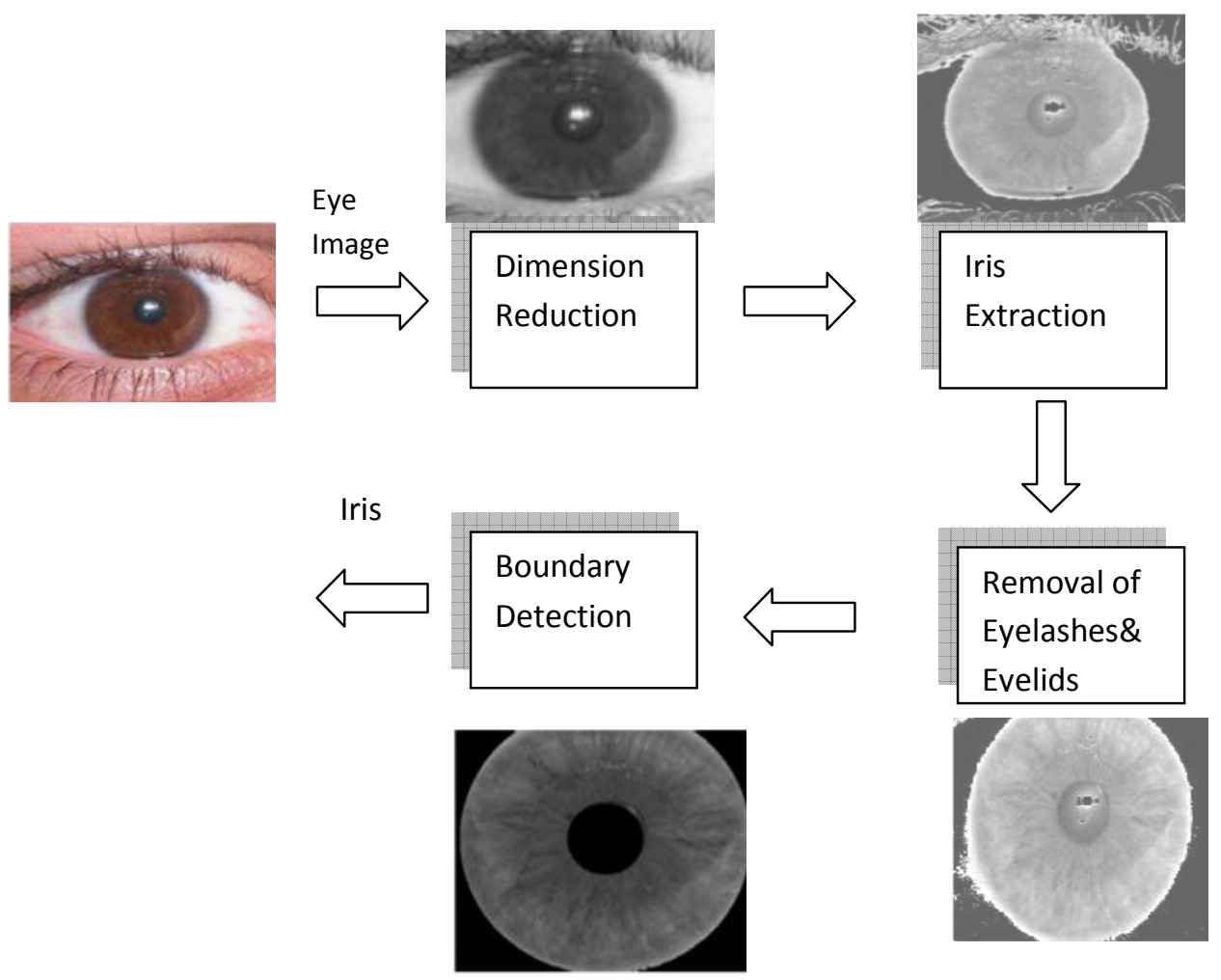

Figure 2 Iris Segmentation Stages

\subsubsection{Dimension Reduction and Iris Extraction}

For the reduction of the computational complexity, the iris images are first converted into gray scale images. For localization, the assumption that summation of the pixel values in the iris region will be less compared to other region is used. This leads to the use of the threshold technique based on the color of the iris. The gray level values of the iris pixels for a dark iris will be lesser when compared to light iris. Two threshold values can be set to determine the iris region using the histogram of the eye image. For creation of the knowledge base, eye images of both the groups have been used so that threshold value has been computed for the extreme cases.

\subsubsection{Removal of eyelids and eyelashes}

Next stage is the removal of unwanted information, such as eyelashes and eyelids which can be done using Sobel operator for detecting the edges. Sobel operator performs a 2D spatial gradient measurement on an image and returns points where there is maximum gradient of image which helps in edge detection as it is characterised by noticeable change in intensity. Sobel operator consists of two $3 \times 3$ masks $G_{x}$ and $G_{y}$ which are given in equation (1) and (2) respectively.

$$
\begin{gathered}
G_{x}=\left[\begin{array}{ccc}
-1 & 0 & 1 \\
-2 & 0 & 2 \\
-1 & 0 & 1
\end{array}\right] \\
G_{y}=\left[\begin{array}{ccc}
1 & 2 & 1 \\
0 & 0 & 0 \\
-1 & -2 & -1
\end{array}\right]
\end{gathered}
$$


The gradient is approximated by applying the masks to the image and computing its magnitude as in equation (3).

$$
G\left[I\left(i_{y} j\right)\right]=\left|G_{x}\right|+\left|G_{y}\right|
$$

To compute the gradient for the pixel of the input image I is given in equation (4).

$$
I\left(i_{i} j\right)=\left[\begin{array}{ccc}
I(t-1 . j-1) & I(t-1, j) & I(t-1, j+1) \\
I\left(i_{j} j-1\right) & I\left(i_{j} j\right) & I(i j+1) \\
I\left(i+1_{j} j-1\right) & i(i+1, j) & I\left(i+1_{x} j+1\right)
\end{array}\right]
$$

$G_{x}$ and $G_{y}$ can be found as in equations (5) and (6). Gradient magnitude at each pixel is found and compared with the threshold to determine, whether it's an edge pixel or not. Sobel operator is less sensitive to noise, due to its large convolution masks.

$$
\begin{aligned}
& C_{x}\left(i_{y} j\right)=\left\{\begin{array}{c}
{\left[I(i-1, j+1)+2 * I(i, j+1)+I\left(i+1_{j} j+1\right)\right]} \\
-\left[I\left(i-1_{j} j-1\right)+2 * I(i, j-1)+I(i+1, j-1)\right]
\end{array}\right. \\
& G_{y}\left(i_{x} j\right)=\left\{\begin{array}{l}
{\left[I\left(i-1_{j} j-1\right)+2 * I\left(i-1_{j} j\right)+I\left(i-1_{j} j+1\right)\right]} \\
-\left[I\left(i+1_{j} j-1\right)+2 * I(i+1, j)+I\left(i+1_{i} j+1\right)\right]
\end{array}\right.
\end{aligned}
$$

\subsubsection{Boundary Detection}

For boundary detection, the centre pixel of the eyelash removed iris image is determined and a circular strip is extracted based on the centre co-ordinates of the pupil. For detecting the inner and outer boundary ( $B_{\text {in or out }}$ ) of the iris, Integro-Differential operator [2] was used. The integrodifferential operator is defined as in equation (7).

$$
D_{\text {in or out }}=\max _{\left(r, x_{\alpha} y_{0}\right)}\left|G_{\sigma}(r) \times \frac{\partial}{\partial r} \oint_{r, x_{0} y_{0}} \frac{I\left(x_{r} y\right)}{2 \pi r} d s\right|
$$

where $I(x, y)$ is the eye image, $\mathrm{r}$ is the radius to search for, $G_{\sigma}(r)$ is a Gaussian smoothing function and $\mathrm{s}$ is the contour of the circle given by $\mathrm{r}, \mathrm{x}_{0}, \mathrm{y}_{0}$. The operator searches for the circular path where there is maximum change in the pixel value, by varying the radius and centre $\mathrm{x}$ and $\mathrm{y}$ position of the circular contour. The operator is applied iteratively in order to attain precise localization. 


\subsection{Iris Normalization and Unwrapping}

Once the iris region has been successfully segmented from an eye image, it is transformed so that it has fixed dimensions in order to allow comparisons. Most normalization techniques are based on transforming iris into polar coordinates, known as unwrapping process. The normalization process will produce iris region, which have the same constant dimensions, so that two photographs of the same iris under different conditions will have same characteristics features. Figure 3 shows the normalised iris image.

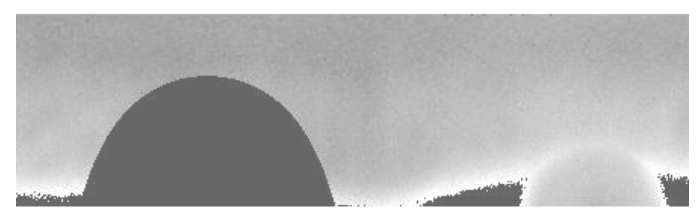

Figure 3. Normalized iris image

In fact homogenous rubber sheet model devised by Daugman remaps each point $(\mathrm{x}, \mathrm{y})$ within the iris region to a pair of polar co-ordinates $(r, \theta)$, where $r$ is on the interval $(0,1)$ and $\theta$ is angle $(0,2 \pi)$. Then the normalized iris region is unwrapped into a rectangular region. Figure 4 illustrates the mechanism of Daugman's rubber sheet model.

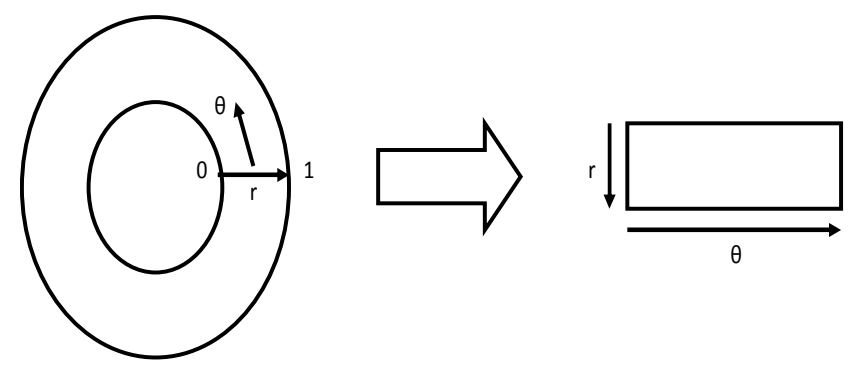

Figure 4. Unwrapping: Daugman's Rubber Sheet Model

The normalized remapping of iris region from cartesian co-ordinates $(\mathrm{x}, \mathrm{y})$ to non-concentric polar representation is modeled as proposed by [7] and is given by equation (8).

$$
I\left(x_{(n, \theta)}, y_{(r, \theta)}\right) \rightarrow I_{(r, \theta)}
$$

where

$$
\begin{aligned}
& x_{(r, \theta)}=(1-r) x_{p}(\theta)+r x_{l}(\theta) \\
& y_{(r, \theta)}=(1-r) y_{p}(\theta)+r y_{l}(\theta)
\end{aligned}
$$

where $I(x, y)$ is the iris region image, $(x, y)$ are the original cartesian co-ordinate, $(r, \theta)$ are the corresponding normalized polar co-ordinates, $\left(x_{p}, y_{p}\right)$ and $\left(x_{l}, y_{l}\right)$ are the pupil and iris boundary respectively along the direction. 


$$
\begin{aligned}
& x_{p}(\theta)=x_{p 0}(\theta)+r_{p} \cos \theta \\
& y_{p}(\theta)=y_{p 0}(\theta)+r_{p} \sin \theta \\
& x_{l}(\theta)=x_{00}(\theta)+r_{l} \cos \theta \\
& y_{l}(\theta)=y_{l 0}(\theta)+r_{l} \sin \theta
\end{aligned}
$$

After getting the normalized polar representation of the iris region, this region is unwrapped by choosing a constant number of points along each radial line, irrespective of how narrow or wide the radius is at a particular angle. Thus, a 2D array is produced with vertical dimensions of radial resolution and horizontal dimension of angular resolution.

\section{Feature EXTRaCtion}

In order to provide accurate recognition of individuals, the most discriminating information present in an iris pattern has been extracted. Only the significant features of the iris have been encoded so that comparison between templates is done.

In the feature extraction stage, first histogram equalization is done to enhance the iris texture in the normalized image. After this, the canny edge detector [8] is used to extract iris texture from the normalized image. This edge detected is a 2D image and hence to reduce the dimension of feature it is converted into a 1D energy signal. Vertical projection is the method used for the conversion from 2D to 1D signal. Discrete wavelet transform is applied to this $1 \mathrm{D}$ energy signal. As a result a set of low frequency and high frequency coefficients are obtained. Since the high frequency coefficients do not contain any information, it is omitted and the low frequency coefficients each of which has a dimension of 64 bytes are taken as the iris templates. The different steps involved in the feature extraction stage are shown in Figure 5.

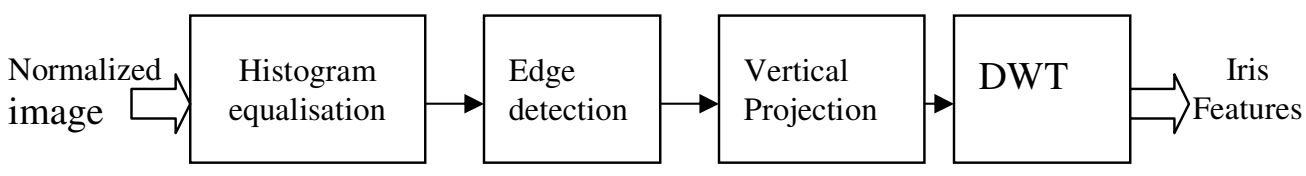

Figure 5. Feature Extraction Stages

\subsection{Histogram Equalization}

This method usually increases the local contrast of many images. Through this adjustment, the intensities can be better distributed on the histogram. This allows for areas of lower local contrast to gain a higher contrast without affecting the global contrast. Histogram equalization accomplishes this by effectively spreading out the most frequent intensity values. The image obtained after histogram equalization is shown in Figure 6. The domes in the unwrapped image are due to the eyelid occlusion.

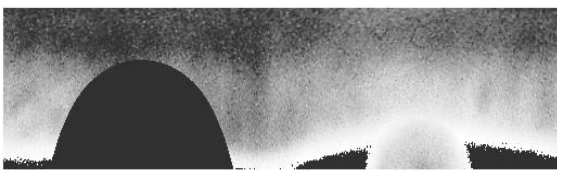

Figure 6. Histogram Equalised Image 


\subsection{Edge Detection}

After histogram equalization iris texture will be enhanced in the normalized image. Edge detection is performed to extract the iris texture from this enhanced image. Though several edge detection techniques such as Sobel, Canny, Prewitt etc. are available, it was observed that Canny edge detection technique is able to extract most of the iris texture from the enhanced image.

The Canny method [9] finds edges by looking for local maxima of the gradient of $I\left(i_{j} j\right)$. The gradient is calculated using the derivative of a Gaussian filter. The method uses two thresholds, to detect strong and weak edges, and include the weak edges in the output only if they are connected to strong edges. This method is therefore less likely than the others to be fooled by noise, and more likely to detect true weak edges. Figure 7 shows the edge detected image using canny operator.

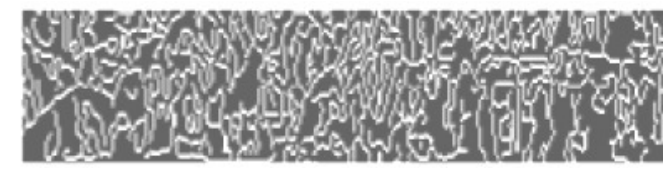

Figure 7. Canny edge detected image

\subsection{Vertical Projection}

Vertical projection is a method used to convert the 2D signal to $1 \mathrm{D}$ signal. This is done to reduce the system complexity. For vertical projection, energy of each row of the edge detected image is calculated and is converted into a row vector. The generalized equation is shown in equation (9). The dimension of normalized image is $m \times n$ and is taken as $128 \times 512$. Hence, after vertical projection its dimension is $\mathrm{m}$, which is equal to 128 .

$$
\left[\begin{array}{ccc}
x_{11} & \ldots & x_{1 n} \\
\vdots & \cdots & \vdots \\
x_{m 1} & \ldots & x_{m n}
\end{array}\right] \rightarrow\left[\begin{array}{lll}
n & & \\
\left|x_{1 i}\right|^{2} & \ldots & \sum_{i=1}^{n}\left|x_{m i}\right|^{2}
\end{array}\right]
$$

\subsection{Discrete Wavelet Transform}

The discrete wavelet transform (DWT) decomposes the signal into mutually orthogonal set of wavelets [10]. The DWT of signal $\mathrm{x}$ is calculated by passing it through a series of filters. First the samples are passed through a low pass filter with impulse response $\mathrm{g}[\mathrm{n}]$ resulting in a convolution given in equation (10).

$$
Y[n]=(x * g)[n]=\sum_{k=-\infty}^{\infty} x[k] g[n-k]
$$

The signal is also decomposed simultaneously using a high pass filter $\mathrm{h}[\mathrm{n}]$. The outputs give the detail coefficients (from the high pass filter) and approximation coefficients (from the low pass filter) whose dimension will be 64 bytes each, since the dimension of $1 \mathrm{D}$ signal is 128 bytes. It is important that two filters are related to each other and they are known as a quadrature mirror filter. Here Haar wavelet [11] is used for wavelet transform. After wavelet transform, a set of low frequency coefficients and high frequency coefficients each of dimension 64 bytes is obtained. After DWT, it is observed that approximation coefficients contain information and detailed 
coefficients do not have any information. Hence approximation coefficients with dimensions 64 bytes is selected as feature vector and stored in database.

\section{Classification}

In recognition stage the features of the input eye image is compared with that of the features that is already stored in the database and if it matches, the corresponding eye image is identified otherwise it remains unidentified. Since a bitwise comparison is necessary Hamming distance was chosen for identification.

\subsection{Hamming Distance}

The Hamming distance [12] gives a measure of how many bits are the same between two bit patterns. It is used for comparison of iris templates in the recognition stage. Hamming distance D is given by equation (11).

$$
D=\frac{1}{n} \sum_{k=1}^{n} x_{k} \oplus y_{k}
$$

where, $\mathrm{x}$ and $\mathrm{y}$ are the two bit patterns of the iris code. $\mathrm{n}$ indicates number of bits. Hamming distance $\mathrm{D}$ gives out the number of disagreeing bits between $\mathrm{x}$ and $\mathrm{y}$.

Ideally, the hamming distance between two iris codes generated for the same iris pattern should be zero; however this will not happen in practice due to fact that normalization is not perfect. The larger the hamming distances (closer to 1), the more the two patterns are different and closer this distance to zero, the more probable the two patterns are identical. By properly choosing the threshold upon which we make the matching decision, one can get good iris recognition results with very low error probability.

\section{RESULTS AND DISCUSSIONS}

The system was tested using the UBIRIS database [5] which included 1877 images from 241 persons collected in two sessions. The images collected in the first photography session were low noise images. On the other hand, images collected in the second session were captured under natural luminosity factor, thus allowing reflections, different contrast levels, and luminosity and focus problems thus making it a good model for realistic situations.

Ten sets of eye images from UBIRIS database was taken for identification. Each set consists of three eye images of a person taken at different time. From each set a single eye image was randomly selected and its features were stored in the database. Therefore a total of 18 images were used for simulation. These images are called registered images since its feature is stored in the knowledge base. The main challenge in the identification is to identify the other two images in each set whose features are not stored. 6 images whose features are not stored in the database are also used to test the algorithm. These images are called as unregistered images. An efficient algorithm should identify all registered images and reject all unregistered images. Performance of iris acceptance algorithm is validated using four parameters -False Reject (FR), False Accept(FA), Correct Reject(CR) and Correct Accept(CA). FR is obviously the case where we judge a pattern as not the target one while it is. FA is when the pattern is considered as the target one while it is not. CR is when the pattern is correctly judged as being not the target one. Finally, $\mathrm{CA}$ is when the pattern is correctly considered to be the targeted one. These outcomes are 
illustrated in Figure 8. It was found that an optimum result is obtained at hamming distance threshold of 0.4 .

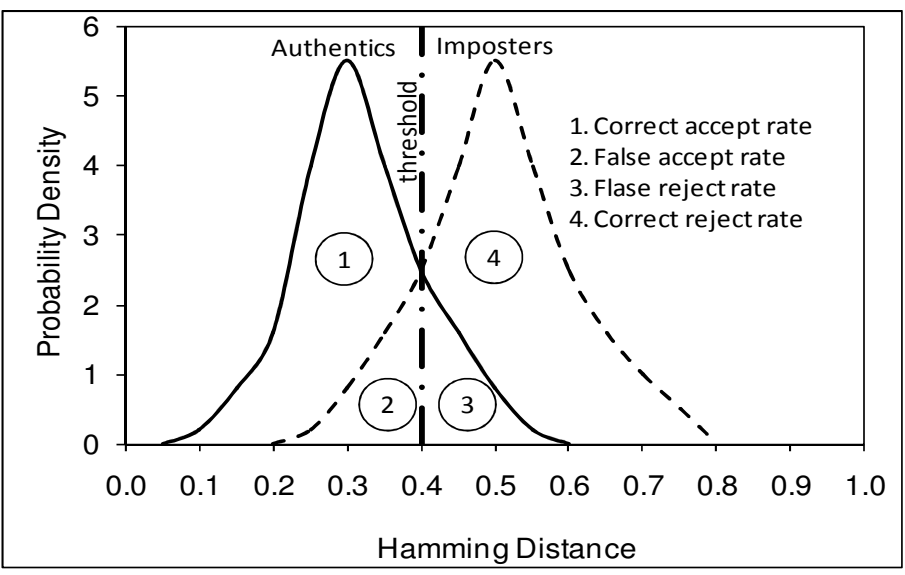

Figure 8. Decision making in iris biometric system

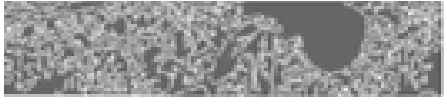

(a) Canny

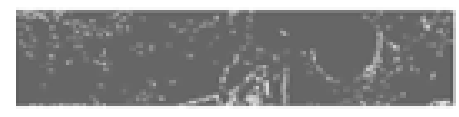

(c)Prewitt

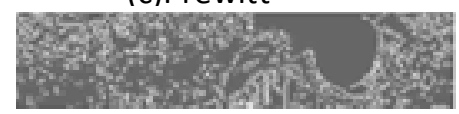

(e) Log
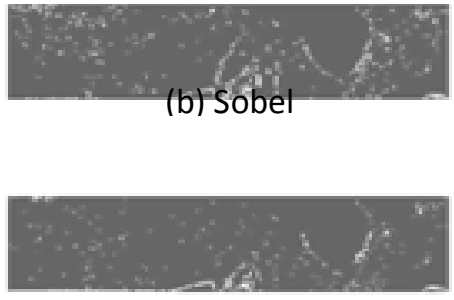

(d) Robert

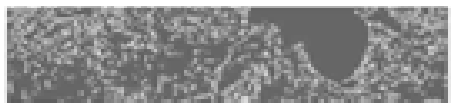

(f)Zero Cross

Figure 9. Detection of iris edge using various operators

Using MATLAB, a comparison study between different classical operators, Canny, Sobel, Prewitt, Roberts, log and zero cross was also done. The operators were applied to the enhanced normalized image. The results, presented in Figure 9, show the performance of each of the operators. It was found that the Canny operator outperforms the others; in fact it was the only operator which was able to extract most of the iris texture.

\section{Conclusions}

Iris images were segmented and projected into 1D signal by the vertical projection and the 1D signal features were extracted by the Haar wavelet transform. The complexity of feature extraction method for iris recognition was low and achieved thus a considerable computational reduction while keeping good performance.

A low dimensional feature extraction algorithm was developed and tested with 24 eye images from the database by varying the parameters such as dimension of feature vector and hamming distance threshold. It was found that an optimum result is obtained at hamming distance threshold of 0.4 and feature vector dimension of 64bytes. It was also observed that canny operator was best 
suited to extract most of the edges to generate the iris code for comparison. Recognition rate of $89 \%$ and rejection rate of $95 \%$ was achieved.

\section{REFERENCES}

[1] Jain A.K, Ross A. and Prabhakar S.(2004) "An Introduction to Biometric Recognition", IEEE Transactions on Circuits and Systems for Video Technology, Special Issue on Image- and VideoBased Biometrics, Vol.14, No.1, pp.4-20.

[2] Daugman J.(2004) "How iris recognition works", IEEE Transactions on Circuits and Systems for Video Technology, Vol.14, No.1, pp.21-30.

[3] Jain A.K, Ross A, Pankanti S,(2006) "Biometrics: A tool for information security", IEEE Transaction on Information Forensics and Security, Vol.1, No.2, pp.125-143.

[4] Ross A, Nandakumar K \& Jain A.K,(2006) "Handbook of Multibiometrics", Springer.

[5] Proenc H, and Alexandre L,(2004) "UBIRIS: Iris Image Database", Available: http://iris.di.ubi.pt.

[6] Zhang D.(2003) "Detecting eyelash and reflection for accurate iris segmentation", International journal of Pattern Recognition and Artificial Intelligence, Vol. 1, No.6, pp.1025-1034..

[7] Jaroslav B, "Circle detection using Hough Transform," Technical Report, University of Bristol, U.K. Available at: http://linux.fjfi.cvut.cz/ pinus/bristol/ imageproc/ hw1/report.pdf

[8] Kovesi P, "MATLAB Function for Computer Vision and Image Analysis", Available at: http://www.cs. uwa. edu.au/ pk/Research/ MatLabFns/ index.html.

[9] Canny J,(1986) “A computational approach to edge detection”, IEEE Transaction on Pattern Analysis and Machine Intelligence, Vol. 8, pp. 679-698.

[10] Boles W, and Boashash B,(1998), "A Human Identification Technique using Images of the Iris and Wavelet Transform”, IEEE Trans. Signal Processing, Vol. 46, No. 4, pp. 1185-1188.

[11] Lim S, Lee K., Byeon O, and Kim T,(2001) "Efficient iris recognition through improvement of feature vector and classifier", ETRI Journal, Vol. 23, No. 2, pp. 61-70.

[12] Daugman J,(1993) "High confidence Visual Recognition of Person by a test of statistical Independence", IEEE transactions on pattern analysis and machine intelligence, Vol.15 No.11, pp. 1148-1161.

\section{Authors}

Binsu C. Kovoor is working as Assistant Professor in Information Technology, Cochin University of Science and Technology 2000 onwards. Her areas of interest include biometric security system, pattern recognition, database systems, data mining, data structures, streaming audio and video signals etc. She is a Life member of ISTE and IE.

Dr. Supriya M. H joined as a faculty in the Department of Electronics, Cochin University of Science \& Technology in 1999. Her fields of interests are Target Identification, Signal Processing, Bioinfomatics, Steganography and Computer Technology. She has presented papers in several International Conferences in Europe, USA, and France. She is actively involved in Research and Development activities in Ocean Electronics and related areas and has a patent and about 87 research publications to her credit. She is a Life member of IETE and ISTE.

Dr. K. Poulose Jacob, Professor of Computer Science at Cochin University of Science and Technology since 1994, is currently Director of the School of Computer Science Studies. His research interests are in Information Systems Engineering, I ntelligent Architectures and Networks.He has more than 90 research publications to his credit. $\mathrm{He}$ has presented papers in several International Conferences in Europe, USA, UK, Australia and other countries. Dr. K. Poulose Jacob is a Professional member of the ACM (Association for Computing Machinery) and a Life Member of the Computer Society of India.
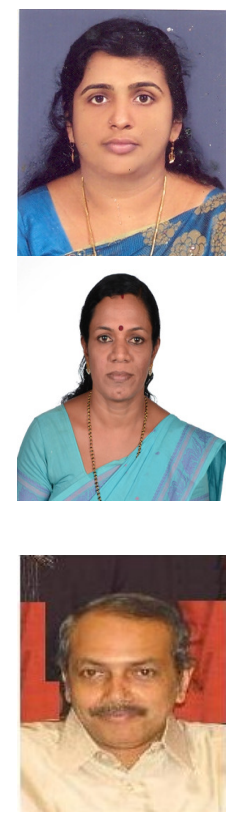